\title{
AN ULTRA WIDEBAND RADAR FOR MICRO AIR VEHICLE APPLICATIONS
}

\author{
Robert J. Fontana, Edward A. Richley, Anthony J. Marzullo, \\ Lance C. Beard, Robert W.T. Mulloy and E.J. Knight
}

Copyright (C) 2002 IEEE. Reprinted from 2002 IEEE Conference on Ultra Wideband Systems and Technologies, May 2002, Baltimore, MD.

This material is posted here with permission of the IEEE. Such permission of the IEEE does not in any way imply IEEE endorsement of any of Multispectral's products or services. Internal or personal use of this material is permitted. However, permission to reprint/republish this material for advertising or promotional purposes or for creating new collective works for resale or redistribution must be obtained from the IEEE by sending a blank email message to pubs-permissions@ieee.org.

By choosing to view this document, you agree to all provisions of the copyright laws protecting it. 


\title{
AN ULTRA WIDEBAND RADAR FOR MICRO AIR VEHICLE APPLICATIONS
}

\author{
Robert J. Fontana, Edward A. Richley, Anthony J. Marzullo, \\ Lance C. Beard, Robert W.T. Mulloy and E.J. Knight \\ Multispectral Solutions, Inc. \\ Germantown, MD USA
}

Tel: +01 301.528.1745 Email: (rfontana,richley,amarzullo,lbeard,rmulloy,ejknight)@multispectral.com

\begin{abstract}
The development and widespread use of micro air vehicles (MAVs) in the battlefield environment has been effectively constrained by the limitations in the MAV sensor suite. Furthermore, the extreme size, weight and power constraints imposed on the on-board electronics for such platforms have made it very difficult to utilize more conventional and commercially available technologies for this application.

This paper discusses the development of an extremely small, micro-power, radar system utilizing ultra wideband (UWB) technology which addresses MAV mission requirements for collision avoidance and precision altimetry in support of autonomous vehicle operation.
\end{abstract}

\section{INTRODUCTION}

Currently, the term "micro air vehicle" or MAV refers to a new class of aircraft whose target dimensions are less than $15 \mathrm{~cm}$ (6 in) in any one dimension. In the future, even insect size vehicles are envisioned. The Defense Advanced Research Projects Agency (DARPA) is the U.S. agency at the forefront of MAV development [1,2]. The military application for MAVs is primarily in the area of reconnaissance, and the projection is for tiny "spy planes" that a soldier can carry in a backpack, launch and use to scout ahead for enemy troops. Dr. Jim McMichael, first DARPA Program Manager for MAV development, envisioned an urban setting for many MAV scenarios [2]:

"In urban operations, MAVs, acting in small cooperative groups, will enable reconnaissance and surveillance of inner city areas, and may serve as communication relays. They may also enable observations through windows, and sensor placement on vertical and elevated surfaces. Their application to building interiors is the most demanding envisioned. The capability to navigate complex shaped passageways, avoid obstacles and relay information will require yet another level of technology.... Constricted corridors of complex geometry, multiple obstacles --and some of them moving - must all be reckoned with if the MAV is to become useful to the warfighter."
Since initial concept definition, numerous other applications have also been proposed which include use in search and rescue; remote nuclear, biological and chemical (NBC) sensing; monitoring of traffic patterns and airborne pollutants, etc. Small size, low cost and low detectability (both visually and electromagnetically) have made such vehicles very attractive to the military.

Response time limitations in human piloting of such vehicles points out the need for fully autonomous flight to support these demanding scenarios. However, in order for MAVs to operate autonomously, a collision avoidance sensor capable of detecting obstructions and hazards to flight is an absolute requirement. Additionally, a fine resolution, short-range altimeter capability is needed to aid these, often fragile, micro vehicles in takeoff and landing, and to assist in hovering for surveillance applications.

As seen above, the envisioned operating environments for MAVs are quite complex and diverse, ranging from urban population centers to extreme wilderness environments. Thus onboard sensor suites must be capable of maintaining a high level of performance in a wide array of scenarios and against very diverse targets. Additionally, the physical constraints of the MAV present significant challenges to the integration of sensor packages onboard the vehicle. Candidate sensors must be extremely lightweight, occupy minimal space onboard the vehicle and must also have very low power consumption.

Ultra wideband (UWB) radar has emerged as a leading technology candidate for MAV applications due to several important technical considerations:

An extremely short duration pulse not only provides for fine radar range resolution (essential for meeting demanding autonomous flight and precision landing requirements); but also results in a low duty cycle waveform which can minimize the prime power demands on the vehicle. For example, a $10 \mathrm{kpps}$ UWB radar operating with a $500 \mathrm{MHz}$ instantaneous bandwidth, has a pulse duty cycle of roughly $2 \times 10^{-5}$. Thus, by time-power gating (see below), the average power drain can be many orders of magnitude smaller than the peak power requirement for the radar.

Furthermore, low duty cycle emissions also result in low average power densities (Watts per unit Hertz) which 
are essential to minimize interference to other onboard electronics - most importantly, the vehicle's flight control system and associated telemetry link. Of course for military operations, a low power spectral density is of importance in making the vehicle less vulnerable to intercept and subsequent electronic countermeasures (ECM) attack.

Another important feature of short pulse technology is the ability to establish precision range gates at user selectable distances. These range gates allow the vehicle radar to eliminate detections outside of selected areas of interest and dramatically reduces the number of nuisance and false alarms. This is of particular advantage in high clutter environments such as urban centers or heavily forested terrain. Additionally, since UWB-based radars function as presence sensors, they do not depend upon relative motion or Doppler information. Thus, they are suitable for a wide variety of operational scenarios including slow moving or hovering platforms.

With its inherently large bandwidth waveform, a UWB radar also provides an enhanced detection probability against complex and low radar cross section (RCS) targets such as suspended wires and utility poles [3]. Finally, since UWB radar designs are nearly all digital, with minimal RF and microwave electronics, low cost microminiaturization is possible through the use of custom application specific integrated circuit (ASIC) and radio frequency integrated circuit (RFIC) technologies. In addition, the commonality of signal generation and processing architectures for both radar and communications permits the design of a multi-function unit that can encompass altimetry and obstacle avoidance as well as data link functions [4].

The rest of the paper will discuss the design, development and initial testing of an ultra wideband radar specifically designed for the MAV.

\section{MAV RADAR SYSTEM REQUIREMENTS}

Under previous advanced development efforts [3], Multispectral Solutions, Inc. (MSSI) has demonstrated the capability of an ultra wideband radar sensor to perform both obstacle detection and precision altimetry operations. However in order to meet the stringent physical and primary power constraints of the MAV, further design refinement was necessary. Given MAV constraints, such issues as operational frequency, antenna design and placement, power management, etc., become critically important. In conjunction with DARPA personnel, MSSI developed specifications for an ultra wideband MAV collision and obstacle avoidance radar:

Weight: Less than 50 grams (10 grams ultimate goal) including antenna (without power supply);

Size: Compatible with MAV vehicle constraints;
Power: Less than 1 Watt;

Range: 50 feet (minimum) for obstacle avoidance;

Resolution: Better than $1 \mathrm{ft} .(30.5 \mathrm{~cm})$ vertical (altimetry) or horizontal (target) resolution; Better than \pm 10 degrees angle-of-arrival (azimuthal) resolution;

Span: Angular coverage of 360 degrees in hover mode and \pm 60 degrees in both azimuth and elevation in translational flight model

Update Rate: $>1000$ updates per second.

Notes:

The radar range requirement of 50 feet is related to the minimum time deemed necessary for the MAV to initiate a collision avoidance maneuver given an air speed of 30 feet per second.

Physical dimensions of the electronics package must be consistent with the payload capacity of candidate MAV airframes.

More recently, MAV development within DARPA has transitioned to the development of somewhat larger, Organic All-Weather Targeting Air Vehicles (OAV) [5]. These unmanned air vehicles are designed for Future Combat System (FCS) direct and indirect weapons system targeting at the small unit level. System developments for the MAV, including the MAV collision avoidance radar, are currently being transitioned to this newer platform.

\section{MAVCAS, A C-BAND UWB RADAR}

$M A V C A S$, a micro air vehicle collision avoidance sensor, has been developed using an unique, spectrally confined ultra wideband waveform approach. The current prototype (Figure 1) utilizes an instantaneous bandwidth of approximately $500 \mathrm{MHz}$ with a design center frequency of $6.35 \mathrm{GHz}$. Both a low power $(25 \mathrm{~mW}$ peak) and a high power $(0.8 \mathrm{~W}$ peak) mode are available. The prototype radar electronics are contained on a dual sided, 8-layer PCB measuring $65 \mathrm{~mm} \times 70 \mathrm{~mm}$. Digital electronics and DC-to-DC converter are configured on one side of the PCB; while the other side contains all RF and microwave components for the radar. The DC-to-DC converter handles an input range of 2.7 to $30 \mathrm{VDC}$ to allow for flexibility during airframe integration. The total package weight of the electronics is 42.5 grams or 1.5 ounces. 


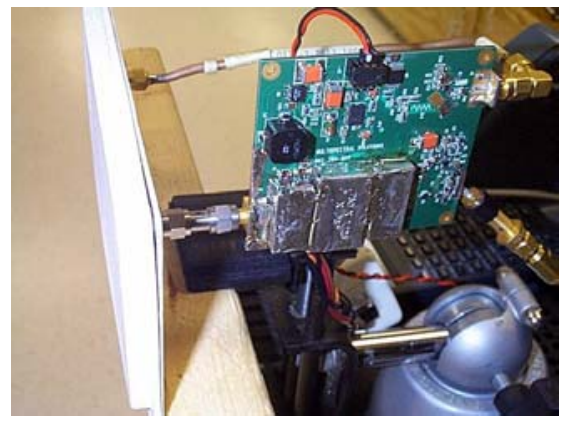

Figure 1. C-band MAVCAS Prototype

Power spectral density and time domain waveforms for MAVCAS are shown below in Figures 3 and 4.

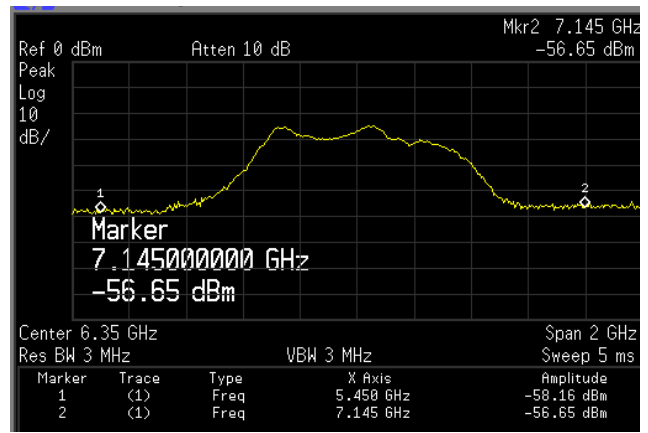

Figure 3. Frequency Domain Response of MAVCAS Transmit Pulse

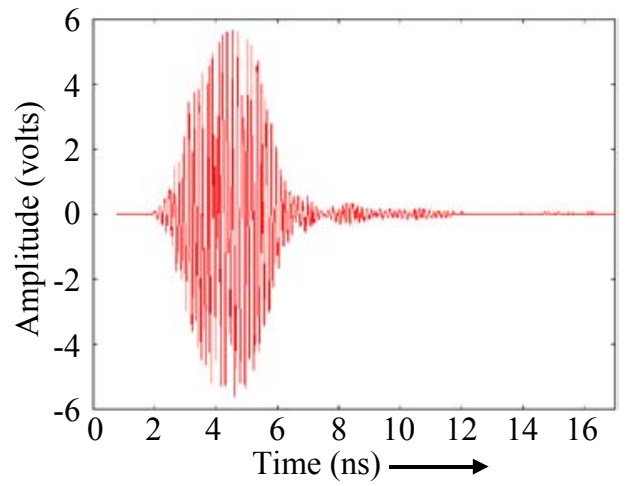

Figure 4. Time Domain Response of MAVCAS Transmit Pulse

Note in Figure 3 that frequency markers are placed near the edges of the FCC Part 15.209 non-restricted band at 5.46 to $7.25 \mathrm{GHz}$, illustrating that the radar response falls entirely within this non-restricted band. Also note, from Figure 4, that the radar is non-coherent (pulse-topulse); and that the pulse envelope is typical of a bandpass filter impulse response. Field strength emission measurements for the radar are discussed further below.

\section{MAVCAS OPERATION}

A system block diagram for the MAVCAS radar sensor is shown in Figure 5 below.

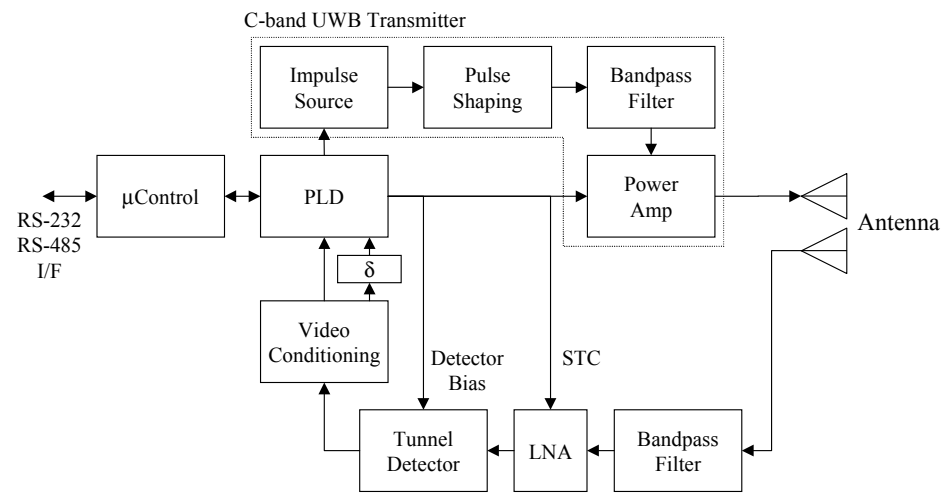

Figure 5. MAVCAS System Block Diagram

MAVCAS system operation proceeds as follows. A programmable logic device (PLD) generates a transmit strobe to the low level impulse source when initiated by a trigger sequence from the system microcontroller. In the low power $(25 \mathrm{~mW})$ mode, the transmit pulse is directly produced by the output of a spectrally filtered, time-gated C-band oscillator; while, in the high power mode, an additional time-gated $\mathrm{C}$-band power amplifier boosts the signal to the $0.8 \mathrm{~W}$ peak power output level [6].

Once the transmitter is triggered, the PLD immediately begins sampling conditioned, baseband (detected) pulses from the receiver tunnel diode detector circuitry. The tunnel diode is preceded by suitable bandpass filtering and an AGC-controlled, low noise amplifier (LNA) which is used to set the system noise temperature.

However, as the PLD clock frequency (in this case, $250 \mathrm{MHz}$ ) is insufficient to achieve a 2 nanosecond (1 foot roundtrip) resolution; the detector output is further subdivided into two separate streams, one of which is delayed by 2 nanoseconds from the other. This is accomplished using a precision, analog delay line which can be implemented in microstrip. Both pulse trains are then clocked into the PLD, and a 2-bit word results which contains information about events occurring on a 2 ns, rather than $4 \mathrm{~ns}$, epoch interval. Note that, in general, the radar resolution can be further improved by adding additional, finer time resolution, delay lines. The radar operates as a presence sensor, determining the distance of an object by simply measuring the roundtrip delay of the transmitted pulse.

Sensitivity time control (STC) [7] for MAVCAS is provided through digital control of both receiver RF gain (RF AGC) and detector bias. RS-232 and RS-485 
interfaces are provided for both user control and data output.

MAVCAS currently utilizes a discrete RF design; however, it is readily adaptable to an RF integrated circuit (RFIC) implementation. This, combined with the replacement of the discrete PLD and microcontroller with a custom application specific integrated circuit (ASIC), are the next steps necessary to fully enable commercial markets and to meet the final size and cost requirements for DARPA's MAV program.

\section{RADIATED EMISSION TESTS}

As shown in the previous section, MAVCAS operates with a tight, spectrally-confined waveform within the Cband region 6.10 to $6.60 \mathrm{GHz}$. Radiated emissions tests have been performed on the radar by an FCC-certified laboratory in the Washington, DC area. An excerpt from this data is illustrated below in Table 1.

These measurements were performed at a peak power output of $0.30 \mathrm{~W}$ (using an earlier MAVCAS RF amplifier design) and with an $18 \mathrm{dBi}$ gain, wideband patch antenna. A picture of the C-band patch array is illustrated in Figure 6 below. Field strength intensity measurements were made at the two FCC restricted band endpoints (5.46 and $7.25 \mathrm{GHz}$ ), at the apparent center frequency of 6.2792 $\mathrm{GHz}$, and at the $2^{\text {nd }}$ and $3^{\text {rd }}$ harmonics of the center frequency (12.5584 and $18.8376 \mathrm{GHz}$, respectively). For these measurements, the pulse repetition frequency (PRF) of the radar was set to its maximum value of $10 \mathrm{kpps}$.

Table 1. MAVCAS Radiated Emissions Measurements
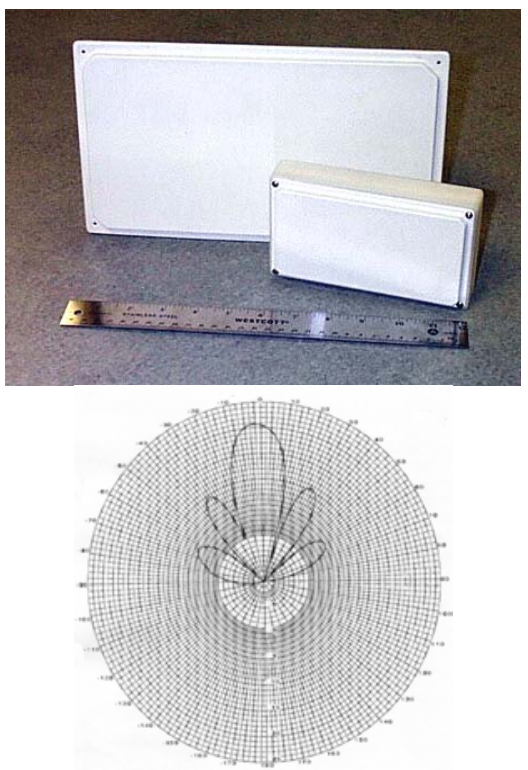

Figure 6. C-band Patch Arrays for MAVCAS

(Top: High gain $+18 \mathrm{dBi}$ and Low gain $+8 \mathrm{dBi}$ designs; Bottom: Pattern for high gain design)

The average E-field for MAVCAS was measured to be $159 \mu \mathrm{V} / \mathrm{m}$ at 3 meters, or $9.9 \mathrm{~dB}$ lower than the FCC Part 15.209 general emissions limit of $500 \mu \mathrm{V} / \mathrm{m}$. Out of band emissions (OOBE) into the nearest restricted bands (i.e., 5.35 to $5.46 \mathrm{GHz}$ on the low side, and 7.25 to 7.75 $\mathrm{GHz}$ on the high side) were not able to be measured above the spectrum analyzer noise floor. In addition, harmonic content of the UWB radar could not be measured within the measurement capability of the spectrum analyzer.

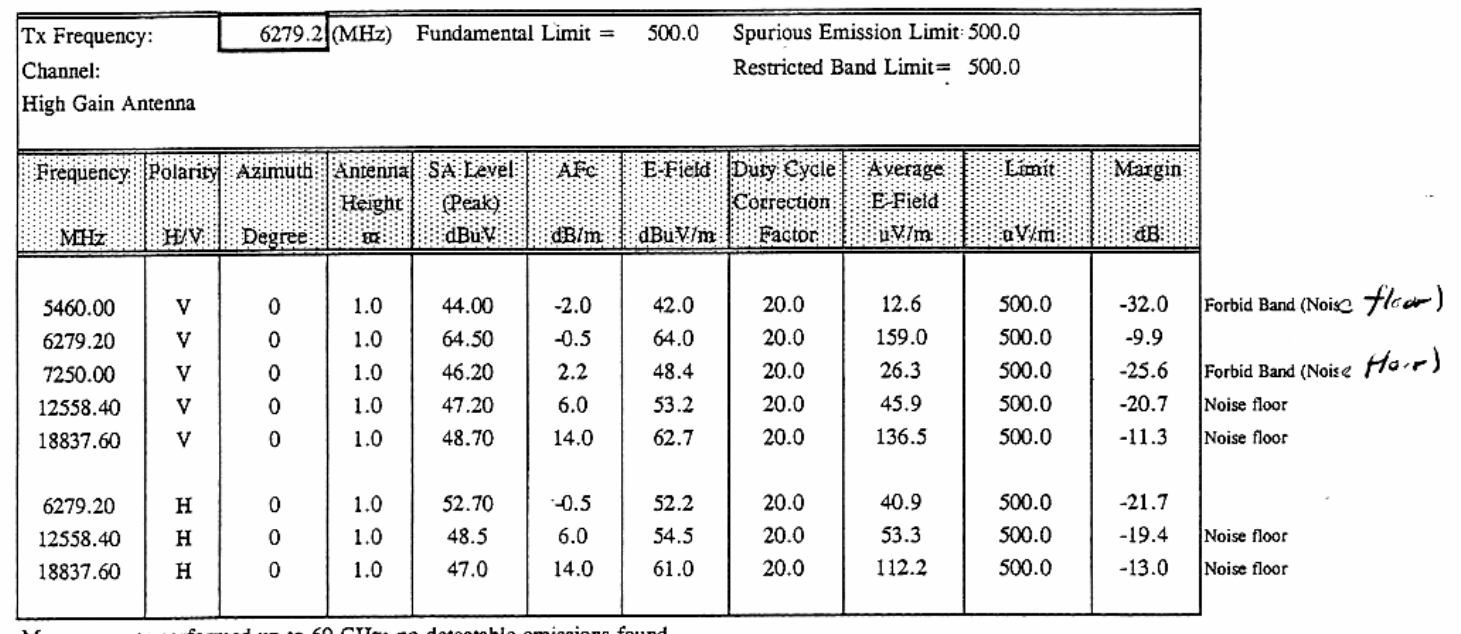

Measurements performed up to $60 \mathrm{GHz}$; no detectable emissions found

\section{PROTOTYPE TESTING}

Initial prototype testing has been completed with the radar in the "low power" mode of operation $(25 \mathrm{~mW}$ peak power). The radar was tested against a variety of targets 
including humans and inanimate objects utilizing the smaller, $+8 \mathrm{dBi}$ dual patch antenna (cf. Figure 6) designed for OAV applications. The prototype achieved a detection range in excess of 70 feet against a typical parking lot lamppost and detected human targets at ranges in excess of 60 feet.

Figure 7 illustrates a typical target return as measured at the output of the radar video detector. In this plot, two radar returns have been captured. The first return is the target of interest, in this case a laboratory chair. The second return is from the laboratory rear wall. Since the speed of light is approximately 0.984 feet per nanosecond $\left(3 \times 10^{8} \mathrm{~m} / \mathrm{s}\right)$, it is evident that the lab chair is located approximately 15 feet from the transmitter $(\sim 30 \mathrm{~ns}$ round trip time), and the back wall is located approximately 38 feet from the transmitter $(\sim 77 \mathrm{~ns}$ round trip time). For the current MAVCAS design, a range resolution of 1 foot was also demonstrated.

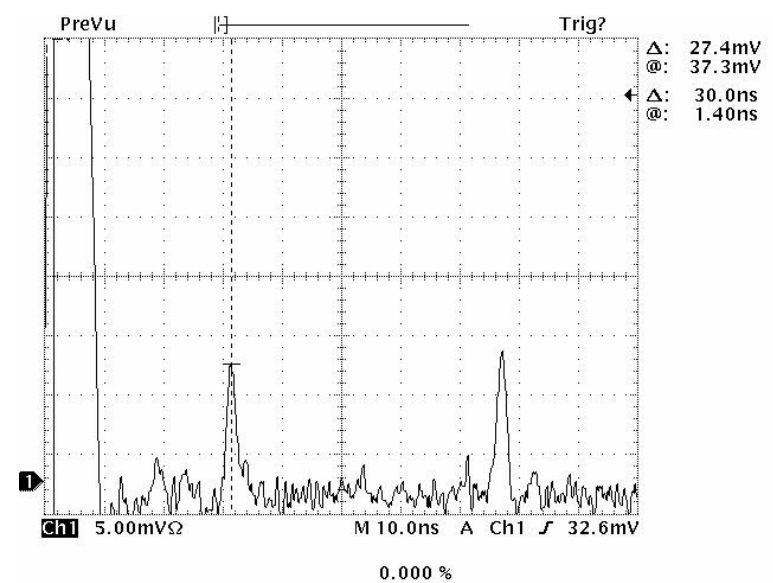

Figure 7. Typical MAVCAS Target Returns (Low Power Mode)

The high power $(0.8 \mathrm{~W})$ mode of operation is intended to allow MAVCAS to be used for precision radar altimetry applications at ranges of up to 1000 feet.

Preliminary testing of the radar as a collision avoidance sensor has been completed; however, testing of the high power mode remains to be completed. System delivery is anticipated in June 2002.

\section{CONCLUSIONS}

Fine range resolution, high power efficiency, low probability of interference and low probability of detection, make UWB an excellent candidate technology for micro and organic air vehicle collision avoidance and altimetry applications. Indeed, micro-power, very small size radars which do not rely upon Doppler, but rather determine the presence or absence of a target, have applicability to a number of commercial problems including collision avoidance sensors for automobiles, perimeter/wide area security and intrusion detection, general aviation applications, etc. To date, more traditional approaches to radar have not enabled large sections of the commercial marketplace due to excessive cost, size, complexity and power.

MAVCAS represents a radical departure from conventional, spectrally inefficient UWB system designs. It represents the first known UWB radar system to be designed to operate entirely within an FCC Part 15 nonrestricted band. As such, it demonstrates that UWB systems can indeed be developed which can readily coexist with existing services.

\section{ACKNOWLEDGMENTS}

This research is supported in part by the Defense Advanced Research Projects Agency under grant DAAH01-00-C-R169.

\section{REFERENCES}

[1] DARPA Tactical Technology Office (TTO), Micro Air Vehicle (MAV) Program; Mr. Sam Wilson, III, Program Manager; URL: www.darpa.mil/tto/programs/mav.html.

[2] McMichael, J.M. and M.S. Francis, "Micro Air Vehicles - Toward a New Dimension in Flight", URL: http://www.darpa.mil/tto/MAV/mav_auvsi.html, August 1997.

[3] Fontana, R.J., J.F. Larrick, J.E. Cade and E. Rivers, "An Ultra Wideband Synthetic Vision Sensor for Airborne Wire Detection", Enhanced and Synthetic Vision 1998, Orlando, FL, April 1998.

[4] Fontana, R.J., "An Ultra Wideband Communication Link for Unmanned Vehicle Applications," Proceedings AUVSI '97, Baltimore, MD, June 3-6, 1997.

[5] DARPA Tactical Technology Office (TTO), Organic Air Vehicle (OAV) Program, Mr. Sam Wilson, III, Program Manager, URL: www.darpa.mil/tto/programs/fcs_oav.html.

[6] Larrick Jr., J.F., R.J. Fontana, U.S. Patent 6,026,125, "Waveform Adaptive Ultra-Wideband Transmitter", February 15, 2000.

[7] Skolnik, M., Radar Handbook, McGraw-Hill, NY, 1990, Chapter 3.6. 